\title{
Extragalactic shocks as cosmic accelerators
}

\author{
Mikhail V. Medvedev \\ Department of Physics and Astronomy, University of Kansas, Lawrence, KS 66045, USA \\ email: medvedev@ku.edu
}

\begin{abstract}
It is quite well established that shocks accelerate particles via the Fermi mechanism. We discuss common features of various extragalactic sources, ranging from Gamma-Ray Bursts and jets of Active Galactic Nuclei to Large-Scale Structure shocks and address how they affect particle acceleration. In particular, we address constraints on the maximum energy of ultrahigh-energy cosmic rays. Interestingly, some recent studies indicate that Fermi acceleration in relativistic shocks (and GRBs, in particular) faces severe difficulties. We will address this issue and demonstrate that the 'observed' shock acceleration of electrons may have nothing to do with Fermi acceleration, but may rather be associated with micro-physics of collisionless shocks.
\end{abstract}

Keywords. cosmic rays, acceleration of particles, shock waves, plasmas, gamma rays: bursts, quasars: general, galaxies: jets, galaxies: clusters: general

\section{General constraints on electromagnetic CR acceleration}

The maximum energy of an accelerated particle (in the absence of any losses) is determined from the confinement argument: the gyro-radius of the particle should not exceed the size of the system, $R$. This sets the maximum energy of the accelerated particle (Hillas 1984), $E_{\text {acc }}=Z e B R \simeq 9.3 \times 10^{23} Z B R_{\mathrm{kpc}} \mathrm{eV}$ where $Z e$ is the charge of a particle, $B$ is the characteristic magnetic field strength in the acceleration region (in gauss), and $R_{\mathrm{kpc}}$ is the size in kiloparsecs. Except for a special case (a rather unnatural one for cosmic ray (CR) sources, such as shocks, jets, supernova, etc.) of the accelerating electric field being parallel to the magnetic field, the confining magnetic field induces a radiation friction force. A particle of very large energy, $E \rightarrow \infty$, traversing a region of size $R$ filled with B-field of strength $B(x)$, will leave the region with energy not exceeding $E_{\mathrm{cr}}=(3 / 2)\left(A m_{p} c^{2} / Z e\right)^{4}\left(\int_{0}^{R} B^{2}(x) d x\right)^{-1} \simeq 2.9 \times 10^{16}(A / Z)^{4} B^{-2} R_{\mathrm{kpc}}^{-1} \mathrm{eV}$ where we assumed that $B \simeq$ constant. We now consider a general case, when a particle is accelerated by an electric field $E_{\text {ind }} \simeq|\mathbf{v} \times \mathbf{B}| / c$ which, in the case of a relativistic system, is thus $\simeq B$. The energy evolution equation reads: $d E / d x \simeq Z e B-(2 / 3)\left(Z e /\left(A m_{p} c^{2}\right)\right)^{4} B^{2} E^{2}$. For a small initial energy of an accelerated particle, the solution of this equation takes a simple and elegant form (Medvedev 2003):

$$
E=\sqrt{E_{\mathrm{acc}} E_{\mathrm{cr}}} \tanh \sqrt{E_{\mathrm{acc}} / E_{\mathrm{cr}}},
$$

where $E$ is the terminal energy of the particle. This solution is plotted in Fig. 1, as a generalized Hillas plot (Medvedev 2003).

\section{Non-Fermi acceleration in GRBs}

Magnetic fields are generated at shocks by the Weibel instability. The fields are associated with current filaments made of protons, whereas the electrons, being much lighter than the protons, are quickly isotropized in the random fields and form a uniform background. Because of uncompensated charge of the filaments, they are also sources of 

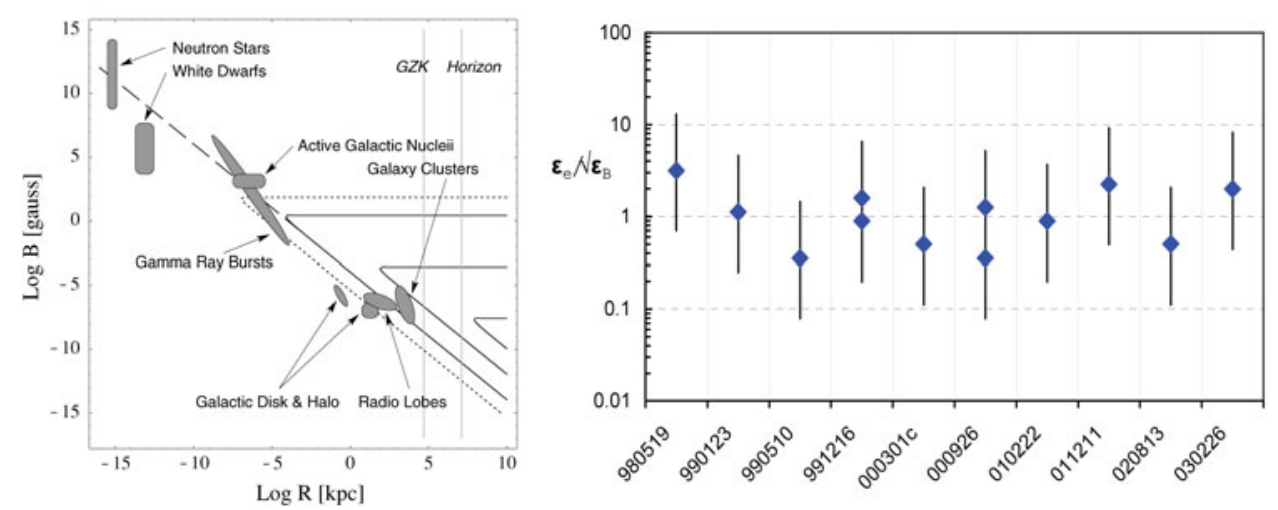

Figure 1. The modified Hillas diagram for UHECR sources. The long-dashed line is the original Hillas relation for a proton of energy $3 \times 10^{20} \mathrm{eV}$. The dotted and solid lines represent the boundaries of the allowed parameter regions from the solution given by Eq. (1.1) for $3 \times 10^{20} \mathrm{eV}$ iron nuclei (dotted line) and for protons of energies $3 \times 10^{20}, 10^{22}$, and $3 \times 10^{23} \mathrm{eV}$, respectively. Only those astronomical objects which fall inside the 'wedges' are, in principle, capable of accelerating the particles to such energies. The gray vertical lines mark two characteristic scales: the GZK attenuation distance $(\sim 20 \mathrm{Mpc})$ and the Hubble horizon size $(\sim 4 \mathrm{Gpc})$.

Figure 2. The $\epsilon_{e} / \sqrt{\epsilon_{B}}$ ratio for ten GRB afterglows analysed by Panaitescu 2005. The parameters of the best fit model (with the lowest $\chi^{2} /$ dof) are used. Clustering of data points around unity is evident.

electrostatic fields. The fields are related to each other as $B=\beta E$, where $\beta=\sqrt{1-\Gamma^{-1}} \simeq 1$. An electron, moving toward a filament gains energy $u_{e} \simeq e l E \simeq e l B$. The typical radial distance the electron travels is comparable to the filament size, which in turn, is of order the relativistic skin depth, $c / \omega_{p p \text {, rel }}$ (here $\omega_{p p, r e l}=\left(4 \pi e^{2} n / m_{p} \gamma_{p}\right)^{1 / 2}$ is the relativistic proton plasma frequency) - the only characteristic scale of the system. Thus $l \simeq \lambda\left(c / \omega_{p p, r e l}\right)$, where $\lambda \simeq 1$ is the dimensionless parameter, which accounts for geometric effects, electrostatic shielding in plasmas, etc. All these effects can introduce a factor of few uncertainty. Finally, the electron energy density behind the shock front is $U_{e}=n u_{e} \simeq \lambda e B n c / \omega_{p p, r e l}$. Once expressed in terms of the equipartition parameters $\epsilon_{B}=B^{2} / 4 \pi /\left(m_{p} c^{2} n \Gamma\right)$ and $\epsilon_{e}=U_{e} /\left(m_{p} c^{2} n \Gamma\right)$ measuring the amount of the total energy of the shock that goes into magnetic fields and the electron heating, the equation for $U_{e}$ takes a simple and elegant form (Medvedev 2007):

$$
\epsilon_{e} \simeq \lambda \sqrt{\epsilon_{B}}
$$

with $\lambda \sim 1$. The value of $\lambda=\epsilon_{e} / \sqrt{\epsilon_{B}}$ derived from spectral fits to GRB afterglows (Panaitescu 2005) is indeed consistent with unity, as our theory predicts, see Fig. 2.

\section{Acknowledgements}

The work is supported by grants DE-FG02-04ER54790 and NNG-04GM41G.

\section{References}

Hillas, A. M. 1984, ARAA, 22, 425

Medvedev, M. V. 2003, PRE, 67, 045401

Medvedev, M. V. 2007, ApJ (Letters), 662, L11

Panaitescu, A. 2005, MNRAS, 366, 1357 